QUARTERLY OF APPLIED MATHEMATICS

VOLUME LXV, NUMBER 1

MARCH 2007, PAGES 189-203

$\mathrm{S} 0033-569 \mathrm{X}(07) 01055-3$

Article electronically published on February 12, 2007

\title{
WELL-POSEDNESS OF TWO-PHASE DARCY FLOW IN 3D
}

\author{
BY \\ DAVID M. AMBROSE \\ Department of Mathematical Sciences, Clemson University, Martin Hall, Clemson, South Carolina \\ 29634
}

\begin{abstract}
We prove the well-posedness, locally in time, of the motion of two fluids flowing according to Darcy's law, separated by a sharp interface in the absence of surface tension. We first reformulate the problem using favorable variables and coordinates. This results in a quasilinear parabolic system. Energy estimates are performed, and these estimates imply that the motion is well-posed for a short time with data in a Sobolev space, as long as a condition is satisfied. This condition essentially says that the more viscous fluid must displace the less viscous fluid. It should be true that small solutions exist for all time; however, this question is not addressed in the present work.
\end{abstract}

1. Introduction. Hele-Shaw flow is best known as a model for the motion of two fluids trapped in a narrow gap between two parallel plates. As such, Hele-Shaw fluids are considered to be two-dimensional. The fluids, rather than satisfying the Navier-Stokes equations, flow according to Darcy's Law, which says that the fluid velocity is proportional to the pressure gradient. While the interpretation of the equations as representing such a fluid flow is necessarily limited to the two-dimensional case, Darcy's Law provides a model for certain fluid flows in three dimensions; there are physical situations in which such a model is relevant. For example, a Darcy flow could arise as a limit of a porous media flow [8, or relating to tumor growth [7, 12, or through the Stefan problem [13]. For further examples, we refer the reader to the references of [9].

This paper proves the well-posedness, locally in time, of a two-phase Darcy flow in three dimensions, without surface tension at the interface. The author has proved the corresponding result for two-dimensional flow in 2]. There, the method used is the same as in 1 to prove well-posedness of a vortex sheet with surface tension in two dimensions. The method makes use of several ideas from the numerical work of Hou, Lowengrub, and Shelley for vortex sheets and Hele-Shaw problems [14, 15. In particular, convenient variables and parameterizations are chosen, and the most important terms are found from the singular integrals in the problem (Hou et al. call this finding a smallscale decomposition). This allows the equations of motion to be written as a semilinear

Received November 16, 2006.

2000 Mathematics Subject Classification. Primary 35Q35.

The author was supported by NSF grant DMS-0610898.

(C)2007 Brown University 
hyperbolic system in the case of the vortex sheet with surface tension, a semilinear parabolic system in the case of Hele-Shaw flow with surface tension, or a quasilinear parabolic system in the case of Hele-Shaw flow without surface tension.

Analytically, once the system has been rewritten in this way, energy estimates can be performed, and the system can then be proved to be well-posed, locally in time, using standard techniques. In two dimensions, the variables used to describe the free surface (the interface between the two fluids), which is a one-dimensional curve, are the tangent angle the curve forms with the horizontal and the arclength. Furthermore, an arclength parameterization is chosen, so that arclength is essentially eliminated from the problem.

In three dimensions, there is no clear, direct analogue of arclength. In the twodimensional case, the tangential velocity of the interface was used to enforce the arclength parameterization. In the three-dimensional case, there are two tangential velocities of the interface, so we may use these to enforce two conditions on the parameterization. This will be described in more detail below; we will say for now that we choose the coordinates to be isothermal (see (2) below). These coordinates have been used in [3] to prove well-posedness of a vortex sheet with surface tension in three dimensions. Such coordinates were chosen for that problem at the suggestion of Jalal Shatah; the author thanks him for several helpful conversations.

Other authors have addressed well-posedness of similar flows. Escher and Simonett have proved existence and uniqueness of solutions for one-phase multi-dimensional HeleShaw flow with surface tension in 9. Chen and Friedman have proven well-posedness for a model which couples a Darcy flow with reaction-diffusion equations, modeling tumor growth, in [7]. Global existence for small data for such problems has been proved by Escher and Simonett for the one-phase or two-phase problem with surface tension [10] and by Friedman and Reitich [13] for the multidimensional Stefan problem with surface tension. In [8], a porous media problem is treated in two and three space dimensions; in this case, the two fluids are taken to have the same viscosity but different densities. In two space dimensions, it has been proved that small solutions to the Hele-Shaw problem without surface tension exist for all time [18.

The plan of the paper is as follows: in Section 2, we provide the formulation of the problem. Since the flow is a potential flow (the velocity in the interior of either fluid is a gradient and is divergence free), it is sufficient to analyze quantities only on the free surface. We explain the variables used to describe the free surface and the parameterization in Section 2. In subsection 2.2, we have a discussion of the BirkhoffRott integral, and how we understand it in relation to Riesz transforms. We begin Section 3 with a few estimates of quantities such as the velocities in terms of the mean curvature of the free surface. Then, we have an energy estimate for the mean curvature. We state our conclusions in Section 4.

We conclude the introduction with a couple of remarks. First, we will be proving an energy estimate with $\kappa$, the mean curvature of the free surface, in the Sobolev space $H^{s}$. We do not determine the minimum $s$ for the argument to work. It is possible that $s \geq 3$ is sufficient; it certainly is the case that $s \geq 6$ is sufficient. As a final introductory remark, we note that the method of the present paper does work when surface tension 
is included. As problems with surface tension have been studied extensively by other authors, we look only at the case without surface tension.

Remark. It is understood that when we say $\mathbf{X} \in H^{k}$, this means that $\mathbf{X}(\alpha, \beta)-$ $(\alpha, \beta, 0)$ is actually in $H^{k}$, since the surface $\mathbf{X}$ is asymptotic to the plane at infinity. This convention applies as appropriate.

2. The formulation of the problem. We consider a free surface between two three-dimensional fluids; the surface is thus two-dimensional and parameterized by the spatial variable $\vec{\alpha}=(\alpha, \beta)$. We write the Cartesian coordinates of the surface as $\mathbf{X}(\vec{\alpha})=$ $(x(\vec{\alpha}), y(\vec{\alpha}), z(\vec{\alpha}))$. We denote the unit tangent and normal vectors by

$$
\hat{\mathbf{t}}^{1}=\frac{\mathbf{X}_{\alpha}}{\left|\mathbf{X}_{\alpha}\right|}, \quad \hat{\mathbf{t}}^{2}=\frac{\mathbf{X}_{\beta}}{\left|\mathbf{X}_{\beta}\right|}, \quad \hat{\mathbf{n}}=\hat{\mathbf{t}}^{1} \times \hat{\mathbf{t}}^{2} .
$$

The velocity of the free surface can be characterized by its normal velocity and by two tangential velocities; that is,

$$
\mathbf{X}_{t}=U \hat{\mathbf{n}}+V_{1} \hat{\mathbf{t}}^{1}+V_{2} \hat{\mathbf{t}}^{2} .
$$

The normal velocity must be chosen according to the fluid dynamics $(U=\mathbf{W} \cdot \hat{\mathbf{n}} ;$ this will be explained below), but the two tangential velocities can be chosen to enforce a choice of parameterization of the surface. If we introduce the first fundamental coefficients of the surface,

$$
E=\mathbf{X}_{\alpha} \cdot \mathbf{X}_{\alpha}, \quad F=\mathbf{X}_{\alpha} \cdot \mathbf{X}_{\beta}, \quad G=\mathbf{X}_{\beta} \cdot \mathbf{X}_{\beta},
$$

then we will use $V_{1}$ and $V_{2}$ to enforce the conditions

$$
E=G, \quad F=0 .
$$

This implies that $V_{1}$ and $V_{2}$ must satisfy

$$
\begin{aligned}
& \left(\frac{V_{1}}{\sqrt{E}}\right)_{\alpha}-\left(\frac{V_{2}}{\sqrt{E}}\right)_{\beta}=\frac{U(L-N)}{E}, \\
& \left(\frac{V_{1}}{\sqrt{E}}\right)_{\beta}+\left(\frac{V_{2}}{\sqrt{E}}\right)_{\beta}=\frac{2 U M}{E} .
\end{aligned}
$$

To be clear, we remark that we define $V_{1}$ and $V_{2}$ to be the unique solution of (3), (4) that decays at infinity.

The choice of the isothermal coordinates above has a beneficial consequence: $E$ is more regular than one might guess at first. If $\mathbf{X} \in H^{k}$, then it turns out that $E \in H^{k}$ also, even though $E$ is defined in terms of first derivatives of $\mathbf{X}$. This is the content of Lemma 4 below.

The free surface can be completely described by its first and second fundamental forms. The first fundamental form is given by $E, F$, and $G$ above. The second fundamental form is given by

$$
L=-\mathbf{X}_{\alpha} \cdot \hat{\mathbf{n}}_{\alpha}, \quad M=-\mathbf{X}_{\alpha} \cdot \hat{\mathbf{n}}_{\beta}=-\mathbf{X}_{\beta} \cdot \hat{\mathbf{n}}_{\alpha}, \quad N=-\mathbf{X}_{\beta} \cdot \hat{\mathbf{n}}_{\beta} .
$$

It is well known that given any six functions, they are not necessarily the first and second fundamental form of any surface; rather, three compatibility conditions must be satisfied. We are furthermore specifying that $E=G$ and $F=0$. Thus, to be able to reconstruct 
the surface, we will only need to know $E$ and some portion of the second fundamental form. In particular, we will evolve the mean curvature of the free surface, which in these coordinates can be expressed as

$$
\kappa=\frac{L+N}{2 E}
$$

We are able to infer evolution equations for $E$ and $\kappa$ from (1). A convenient form of the evolution equation for $\kappa$ is

$$
\begin{aligned}
(\sqrt{E} \kappa)_{t}=\frac{1}{2 \sqrt{E}} \Delta U+\frac{V_{1}}{\sqrt{E}}(\sqrt{E} \kappa)_{\alpha} & +\frac{V_{2}}{\sqrt{E}}(\sqrt{E} \kappa)_{\beta} \\
& +\frac{U M^{2}}{\sqrt{E}}+\frac{L}{2 \sqrt{E}}\left(\frac{V_{1}}{\sqrt{E}}\right)_{\alpha}+\frac{N}{2 \sqrt{E}}\left(\frac{V_{2}}{\sqrt{E}}\right)_{\beta} .
\end{aligned}
$$

2.1. The $\mu$ equation. In each phase, we have the velocity

$$
\mathbf{v}_{i}=-\frac{b^{2}}{12 \nu_{i}} \nabla\left(p_{i}+\rho_{i} g z\right)
$$

The potential in each phase is then

$$
\phi_{i}=-\frac{b^{2}}{12 \nu_{i}}\left(p_{i}+\rho_{i} g z\right)
$$

Since the fluids are incompressible $\left(\operatorname{div}\left(\mathbf{v}_{i}\right)=0\right)$, we see that $\Delta \phi^{i}=0$. Thus, we can write $\phi^{i}$ using a double-layer potential representation with source strength $\mu$ :

$$
\phi_{i}(x, y, z)= \pm \frac{1}{2 \pi} \iint \mu(\alpha, \beta) \frac{(x, y, z)-(x(\alpha, \beta), y(\alpha, \beta), z(\alpha, \beta))}{|(x, y, z)-(x(\alpha, \beta), y(\alpha, \beta), z(\alpha, \beta))|^{2}} \cdot \hat{\mathbf{n}}(\alpha, \beta) d \alpha d \beta \text {. }
$$

The difference between $\phi_{1}$ and $\phi_{2}$ at the free surface is $\mu$ :

$$
\mu=\frac{b^{2}}{12}\left(-\frac{p_{1}}{\nu_{1}}+\frac{p_{2}}{\nu_{2}}\right)-\frac{b^{2}}{12}\left(\frac{\rho_{1} g z}{\nu_{1}}-\frac{\rho_{2} g z}{\nu_{2}}\right)
$$

We will also be interested in the sum of the two potentials at the free surface:

$$
\phi_{1}+\phi_{2}=\frac{b^{2}}{12}\left(-\frac{p_{1}}{\nu_{1}}-\frac{p_{2}}{\nu_{2}}\right)-\frac{b^{2}}{12}\left(\frac{\rho_{1} g z}{\nu_{1}}+\frac{\rho_{2} g z}{\nu_{2}}\right) .
$$

Solving (7) and (8) for $p_{1}$ and $p_{2}$ yields

$$
\begin{aligned}
& p_{1}=-\frac{6 \nu_{1}}{b^{2}}\left(\mu+\left(\phi_{1}+\phi_{2}\right)\right)-\rho_{1} g z, \\
& p_{2}=-\frac{6 \nu_{2}}{b^{2}}\left(-\mu+\left(\phi_{1}+\phi_{2}\right)\right)-\rho_{2} g z .
\end{aligned}
$$

The boundary condition (in the absence of surface tension) is $p_{1}=p_{2}$. Thus, solving (9) and (10) for $\mu$, we have

$$
\mu=\frac{\nu_{2}-\nu_{1}}{\nu_{1}+\nu_{2}}\left(\phi_{1}+\phi_{2}\right)+\frac{b^{2}\left(\rho_{2}-\rho_{1}\right) g}{6\left(\nu_{2}+\nu_{1}\right)} z
$$

[Notice that (11) is an integral equation for $\mu$, since we can write $\phi_{1}+\phi_{2}$ with the doublelayer potential representation (6) above. To write (11) explicitly as an integral equation, we would just need to take the limit in (6) at the free surface. A version of the same integral equation appears in many works in the area of free-surface flows, for example, [19]. We briefly discuss the solvability of the integral equation in Lemma 6 below.] 
We use the names $A_{\nu}=\left(\nu_{1}-\nu_{2}\right) /\left(\nu_{1}+\nu_{2}\right)$ and $R=\frac{b^{2}\left(\rho_{1}-\rho_{2}\right) g}{6\left(\nu_{1}+\nu_{2}\right)}$. Taking a derivative of (11) with respect to $\alpha$, we get

$$
\mu_{\alpha}=-A_{\nu}\left(\nabla \phi_{1} \cdot \mathbf{X}_{\alpha}+\nabla \phi_{2} \cdot \mathbf{X}_{\alpha}\right)-R z_{\alpha} .
$$

The limiting values of the velocities can be found with the Plemelj formulas; see [6] for a discussion. These limiting values are

$$
\nabla \phi_{i}=\mathbf{W} \pm \frac{\mu_{\alpha}}{2 \sqrt{E}} \hat{\mathbf{t}}^{1} \pm \frac{\mu_{\beta}}{2 \sqrt{E}} \hat{\mathbf{t}}^{2},
$$

where the plus signs are taken for $i=1$ and the minus signs are taken for $i=2$. The Birkhoff-Rott integral, W, will be defined and discussed in the next section. Thus, our formula for $\nabla_{\vec{\alpha}} \mu$ is

$$
\begin{aligned}
& \mu_{\alpha}=-2 A_{\nu} \sqrt{E} \mathbf{W} \cdot \hat{\mathbf{t}}^{1}-R z_{\alpha}, \\
& \mu_{\beta}=-2 A_{\nu} \sqrt{E} \mathbf{W} \cdot \hat{\mathbf{t}}^{2}-R z_{\beta} .
\end{aligned}
$$

We will later obtain the quantities $\mu_{\alpha \alpha}$ and $\mu_{\beta \beta}$, so we will need expressions for derivatives of $z_{\alpha}$ and $z_{\beta}$ in terms of $\kappa$ (or at least in terms of $L, M$, and $N$ ). We begin with $z_{\alpha}$. Recall that $\hat{\mathbf{t}}^{1}=\frac{1}{\sqrt{E}}\left(x_{\alpha}, y_{\alpha}, z_{\alpha}\right)$. So,

$$
z_{\alpha \alpha}=\left(\sqrt{E} \hat{\mathbf{t}}^{1} \cdot(0,0,1)\right)_{\alpha} .
$$

Applying the derivative on the right-hand side, we have

$$
z_{\alpha \alpha}=\frac{E_{\alpha} z_{\alpha}}{2 E}+\sqrt{E}\left(\hat{\mathbf{t}}_{\alpha}^{1} \cdot \hat{\mathbf{n}}\right)(\hat{\mathbf{n}} \cdot(0,0,1))+\sqrt{E}\left(\hat{\mathbf{t}}_{\alpha}^{1} \cdot \hat{\mathbf{t}}^{2}\right)\left(\hat{\mathbf{t}}^{2} \cdot(0,0,1)\right) .
$$

We use the name $h=\hat{\mathbf{n}} \cdot(0,0,1)=\frac{1}{E}\left(x_{\alpha} y_{\beta}-y_{\alpha} x_{\beta}\right)$. We now have

$$
z_{\alpha \alpha}=\frac{E_{\alpha} z_{\alpha}}{2 E}-\frac{E_{\beta} z_{\beta}}{2 E}+L h
$$

In the same fashion, we are able to find the following formula for $z_{\beta \beta}$ :

$$
z_{\beta \beta}=\frac{E_{\beta} z_{\beta}}{2 E}-\frac{E_{\alpha} z_{\alpha}}{2 E}+N h .
$$

2.2. The Birkhoff-Rott integral. As usual, we can recover velocity from vorticity. The fluids under consideration are irrotational in the interior of the fluid regions, but there is a jump in velocity across the free surface. This implies that we have a measure-valued vorticity, supported only on the free surface. Thus, we are led to a singular integral (integrating over the free surface) for the velocity. It is the Birkhoff-Rott integral, which in the case of three-dimensional fluids is

$$
\mathbf{W}(\vec{\alpha})=-\frac{1}{4 \pi} \mathrm{PV} \iint\left(\mu_{\alpha}^{\prime} \mathbf{X}_{\beta}^{\prime}-\mu_{\beta}^{\prime} \mathbf{X}_{\alpha}^{\prime}\right) \times \frac{\mathbf{X}-\mathbf{X}^{\prime}}{\left|\mathbf{X}-\mathbf{X}^{\prime}\right|^{3}} d \overrightarrow{\alpha^{\prime}},
$$

where variables followed by a prime are evaluated at $\vec{\alpha}^{\prime}=\left(\alpha^{\prime}, \beta^{\prime}\right)$ and variables not followed by a prime are evaluated at $\vec{\alpha}=(\alpha, \beta)$.

We approximate $\mathbf{W}$ in order to understand it better. In particular, we will understand $\mathbf{W}$ as being a sum of some Riesz transforms plus some smooth terms. To this end, we 
now introduce the Riesz transforms. They are the operators $H_{1}$ and $H_{2}$, defined by

$$
\begin{aligned}
& H_{1} f(\vec{\alpha})=\frac{1}{2 \pi} \mathrm{PV} \iint \frac{f\left(\vec{\alpha}^{\prime}\right)\left(\alpha-\alpha^{\prime}\right)}{\left|\vec{\alpha}-\vec{\alpha}^{\prime}\right|^{3}} d \vec{\alpha}^{\prime}, \\
& H_{2} f(\vec{\alpha})=\frac{1}{2 \pi} \operatorname{PV} \iint \frac{f\left(\vec{\alpha}^{\prime}\right)\left(\beta-\beta^{\prime}\right)}{\left|\vec{\alpha}-\vec{\alpha}^{\prime}\right|^{3}} d \vec{\alpha}^{\prime} .
\end{aligned}
$$

We will also need the operator $\Lambda$, defined as $\Lambda=H_{1} D_{\alpha}+H_{2} D_{\beta}$. If we take $\xi=\left(\xi_{1}, \xi_{2}\right)$, then the symbols of these operators are

$$
\hat{H}_{1}(\xi)=\frac{-i \xi_{1}}{|\xi|}, \quad \hat{H}_{2}(\xi)=\frac{-i \xi_{2}}{|\xi|}, \quad \hat{\Lambda}(\xi)=|\xi| .
$$

A couple of important properties of the Riesz transforms are

$$
\left(H_{1}^{2}+H_{2}^{2}\right) f=-f, \quad H_{1} D_{\beta} f=H_{2} D_{\alpha} f .
$$

(The first identity above requires $\hat{f}(0)=0$.) Important properties of $\Lambda$ include the fact that $\Lambda$ is selfadjoint and that

$$
\left(\int f^{2}+f \Lambda f\right)^{1 / 2}
$$

is equivalent to $\|f\|_{1 / 2}$.

We introduce three more operators, $\mathcal{K}[\mathbf{X}], \mathcal{J}[\mathbf{X}]$, and $\mathcal{J}_{1}[\mathbf{X}]$. They are

$$
\begin{aligned}
\mathcal{K}[\mathbf{X}] \mathcal{F}(\vec{\alpha}) & =\frac{1}{4 \pi} \mathrm{PV} \iint \mathcal{F}\left(\vec{\alpha}^{\prime}\right) \times K\left(\vec{\alpha}, \vec{\alpha}^{\prime}\right) d \vec{\alpha}^{\prime}, \\
\mathcal{J}[\mathbf{X}] \mathcal{F}(\vec{\alpha}) & =\frac{1}{4 \pi} \mathrm{PV} \iint \mathcal{F}\left(\vec{\alpha}^{\prime}\right) \times J\left(\vec{\alpha}, \vec{\alpha}^{\prime}\right) d \vec{\alpha}^{\prime}, \\
\mathcal{J}_{1}[\mathbf{X}] \mathcal{F}(\vec{\alpha}) & =\frac{1}{4 \pi} \mathrm{PV} \iint \mathcal{F}\left(\vec{\alpha}^{\prime}\right) \times J_{1}\left(\vec{\alpha}, \vec{\alpha}^{\prime}\right) d \vec{\alpha}^{\prime} .
\end{aligned}
$$

The kernels $K, J$, and $J_{1}$ are given by

$$
\begin{gathered}
K\left(\vec{\alpha}, \vec{\alpha}^{\prime}\right)=\frac{\mathbf{X}-\mathbf{X}^{\prime}}{\left|\mathbf{X}-\mathbf{X}^{\prime}\right|^{3}}-\frac{\mathbf{X}_{\alpha}^{\prime}\left(\alpha-\alpha^{\prime}\right)+\mathbf{X}_{\beta}^{\prime}\left(\beta-\beta^{\prime}\right)}{E^{\prime 3 / 2}\left|\vec{\alpha}-\vec{\alpha}^{\prime}\right|^{3}} \\
-\frac{\frac{1}{2} \mathbf{X}_{\alpha \alpha}^{\prime}\left(\alpha-\alpha^{\prime}\right)^{2}+\frac{1}{2} \mathbf{X}_{\beta \beta}^{\prime}\left(\beta-\beta^{\prime}\right)^{2}+\mathbf{X}_{\alpha \beta}\left(\alpha-\alpha^{\prime}\right)\left(\beta-\beta^{\prime}\right)}{E^{\prime 3 / 2}\left|\vec{\alpha}-\vec{\alpha}^{\prime}\right|^{3}} \\
+\frac{3}{4} \frac{\left(E_{\alpha^{\prime}}\left(\alpha-\alpha^{\prime}\right)+E_{\beta}^{\prime}\left(\beta-\beta^{\prime}\right)\right)\left(\mathbf{X}_{\alpha}^{\prime}\left(\alpha-\alpha^{\prime}\right)+\mathbf{X}_{\beta}^{\prime}\left(\beta-\beta^{\prime}\right)\right)}{E^{\prime 5 / 2}\left|\vec{\alpha}-\vec{\alpha}^{\prime}\right|^{3}}, \\
J\left(\vec{\alpha}, \vec{\alpha}^{\prime}\right)=\frac{\mathbf{X}-\mathbf{X}^{\prime}}{\left|\mathbf{X}-\mathbf{X}^{\prime}\right|^{3}}-\frac{\mathbf{X}_{\alpha}^{\prime}\left(\alpha-\alpha^{\prime}\right)+\mathbf{X}_{\beta}^{\prime}\left(\beta-\beta^{\prime}\right)}{E^{\prime 3 / 2}\left|\vec{\alpha}-\vec{\alpha}^{\prime}\right|^{3}}, \\
J_{1}\left(\vec{\alpha}, \vec{\alpha}^{\prime}\right)=\left(D_{\alpha}+D_{\alpha^{\prime}}\right) J .
\end{gathered}
$$

We can now use these operators to understand $\mathbf{W}$ better. We introduce the notation $g=\mu_{\beta} \mathbf{X}_{\alpha}-\mu_{\alpha} \mathbf{X}_{\beta}$. Simply by adding and subtracting, we can write $\mathbf{W}$ as

$$
\mathbf{W}=H_{1}\left(\frac{g \times \mathbf{X}_{\alpha}}{2 E^{3 / 2}}\right)+H_{2}\left(\frac{g \times \mathbf{X}_{\beta}}{2 E^{3 / 2}}\right)+\mathcal{J}[\mathbf{X}] g .
$$


Carrying out the cross product, this is the same as

$$
\mathbf{W}=H_{1}\left(\frac{\mu_{\alpha}}{2 E^{1 / 2}} \hat{\mathbf{n}}\right)+H_{2}\left(\frac{\mu_{\beta}}{2 E^{1 / 2}} \hat{\mathbf{n}}\right)+\mathcal{J}[\mathbf{X}] g .
$$

We introduce the notation $O\left(H^{k}\right)$; this will denote any function whose norm in $H^{k}$ can be bounded by $\|\mathbf{X}\|_{s+2}$. In particular, we will prove in Section 3.1 that $E$ and $\mu$ are of the form $O\left(H^{s+2}\right)$.

We take an $\alpha$ derivative of $\mathbf{W}$, yielding

$$
\begin{aligned}
\mathbf{W}_{\alpha}=H_{1}\left(\frac{\mu_{\alpha \alpha}}{2 E^{1 / 2}} \hat{\mathbf{n}}\right) & +H_{2}\left(\frac{\mu_{\alpha \beta}}{2 E^{1 / 2}} \hat{\mathbf{n}}\right) \\
& +H_{1}\left(\frac{\mu_{\alpha}}{2 E^{1 / 2}} \hat{\mathbf{n}}_{\alpha}\right)+H_{2}\left(\frac{\mu_{\beta}}{2 E^{1 / 2}} \hat{\mathbf{n}}_{\alpha}\right)+D_{\alpha} \mathcal{J}[\mathbf{X}] g+O\left(H^{s+1}\right) .
\end{aligned}
$$

The terms which we have included in the $O\left(H^{s+1}\right)$ term above are those which have derivatives on $E$. We use the geometric identity

$$
\hat{\mathbf{n}}_{\alpha}=-\frac{L}{E^{1 / 2}} \hat{\mathbf{t}}^{1}-\frac{M}{E^{1 / 2}} \hat{\mathbf{t}}^{2} .
$$

We also pull the vectors outside of the Riesz transforms, incurring only a smooth commutator. That the commutator is smoothing is the content of Lemma 3 (of Section 3.1) below. We have

$$
\begin{aligned}
\mathbf{W}_{\alpha} & =H_{1}\left(\frac{\mu_{\alpha \alpha}}{2 E^{1 / 2}}\right) \hat{\mathbf{n}}+H_{2}\left(\frac{\mu_{\alpha \beta}}{2 E^{1 / 2}}\right) \hat{\mathbf{n}}-H_{1}\left(\frac{\mu_{\alpha} L}{2 E}\right) \hat{\mathbf{t}}^{1} \\
& -H_{1}\left(\frac{\mu_{\alpha} M}{2 E}\right) \hat{\mathbf{t}}^{2}-H_{2}\left(\frac{\mu_{\beta} L}{2 E}\right) \hat{\mathbf{t}}^{1}-H_{2}\left(\frac{\mu_{\beta} M}{2 E}\right) \hat{\mathbf{t}}^{2}+D_{\alpha} \mathcal{J}[\mathbf{X}] g+O\left(H^{s+1}\right) .
\end{aligned}
$$

Furthermore, we can prove that $D_{\alpha} \mathcal{J}[\mathbf{X}] g$ is actually also of the form $O\left(H^{s+1}\right)$. To begin, we notice that (by integrating by parts), we can write

$$
D_{\alpha} \mathcal{J}[\mathbf{X}] g=\mathcal{J}[\mathbf{X}]\left(D_{\alpha} g\right)+\mathcal{J}_{1}[\mathbf{X}](g),
$$

where $\mathcal{J}_{1}[\mathbf{X}]$ is the integral operator defined above. Lemma2 2 below implies that $\mathcal{J}_{1}[\mathbf{X}](g)$ is in fact in $H^{s+1}$. We thus only need to show that $\mathcal{J}[\mathbf{X}]\left(D_{\alpha} g\right)$ is also $O\left(H^{s+1}\right)$. To this end, we write

$$
\begin{aligned}
4 \pi J=4 \pi K+\frac{\frac{1}{2} \mathbf{X}_{\alpha \alpha}^{\prime}\left(\alpha-\alpha^{\prime}\right)^{2}+\frac{1}{2} \mathbf{X}_{\beta \beta}^{\prime}\left(\beta-\beta^{\prime}\right)^{2}+\mathbf{X}_{\alpha \beta}^{\prime}\left(\alpha-\alpha^{\prime}\right)\left(\beta-\beta^{\prime}\right)}{E^{\prime 3 / 2}\left|\vec{\alpha}-\overrightarrow{\alpha^{\prime}}\right|^{3}} \\
-\frac{3}{4} \frac{\left(E_{\alpha}^{\prime}\left(\alpha-\alpha^{\prime}\right)+E_{\beta}^{\prime}\left(\beta-\beta^{\prime}\right)\right)\left(\mathbf{X}_{\alpha}^{\prime}\left(\alpha-\alpha^{\prime}\right)+\mathbf{X}_{\beta}^{\prime}\left(\beta-\beta^{\prime}\right)\right)}{E^{\prime 5 / 2}\left|\vec{\alpha}-\vec{\alpha}^{\prime}\right|^{3}} .
\end{aligned}
$$

The terms with fractions on the right-hand side of (19) lead to terms in $H^{s+1}$, since the associated integral operators are smoothing by one derivative. That $\mathcal{K}[\mathbf{X}]\left(D_{\alpha} g\right)$ is in $H^{s+1}$ is implied by Lemma1 1 below. This proves that $D_{\alpha} \mathcal{J}[\mathbf{X}](g)$ is of the form $O\left(H^{s+1}\right)$.

Lemma 1. If $\mathbf{X} \in H^{s+2}$ and $\mathcal{F} \in H^{s-1 / 2}$, then $\mathcal{K}[\mathbf{X}] \mathcal{F}$ is in $H^{s+1}$, with

$$
\|\mathcal{K}[\mathbf{X}] \mathcal{F}\|_{s+1} \leq c\left(1+\|\mathbf{X}\|_{s+2}\right)^{2}\|\mathcal{F}\|_{s-1 / 2} .
$$

Lemma 2. If $\mathbf{X} \in H^{s+2}$ and $\mathcal{F} \in H^{s+1 / 2}$, then $\mathcal{J}_{1}[\mathbf{X}] \mathcal{F}$ is in $H^{s+1}$, with the estimate

$$
\left\|\mathcal{J}_{1}[\mathbf{X}] \mathcal{F}\right\|_{s+1} \leq c\left(1+\|\mathbf{X}\|_{s+2}\right)^{2}\|\mathcal{F}\|_{s+1 / 2} .
$$


We do not prove Lemma 1 or Lemma 2 here. Instead, we refer the reader to 3 for details.

We are left with the following formulas for the tangential components of $\nabla W$ :

$$
\begin{aligned}
& \mathbf{W}_{\alpha} \cdot \hat{\mathbf{t}}^{1}=-H_{1}\left(\frac{\mu_{\alpha} L}{2 E}\right)-H_{2}\left(\frac{\mu_{\beta} L}{2 E}\right)+O\left(H^{s+1}\right), \\
& \mathbf{W}_{\alpha} \cdot \hat{\mathbf{t}}^{2}=-H_{1}\left(\frac{\mu_{\alpha} M}{2 E}\right)-H_{2}\left(\frac{\mu_{\beta} M}{2 E}\right)+O\left(H^{s+1}\right), \\
& \mathbf{W}_{\beta} \cdot \hat{\mathbf{t}}^{1}=-H_{1}\left(\frac{\mu_{\alpha} M}{2 E}\right)-H_{2}\left(\frac{\mu_{\beta} M}{2 E}\right)+O\left(H^{s+1}\right), \\
& \mathbf{W}_{\beta} \cdot \hat{\mathbf{t}}^{2}=-H_{1}\left(\frac{\mu_{\alpha} N}{2 E}\right)-H_{2}\left(\frac{\mu_{\beta} N}{2 E}\right)+O\left(H^{s+1}\right) .
\end{aligned}
$$

We also have the following formula involving the normal component of $\nabla W$ :

$$
\left(\mathbf{W}_{\alpha} \cdot \hat{\mathbf{n}}\right)_{\alpha}+\left(\mathbf{W}_{\beta} \cdot \hat{\mathbf{n}}\right)_{\beta}=\frac{1}{2} \Lambda\left(\frac{\mu_{\alpha \alpha}+\mu_{\beta \beta}}{\sqrt{E}}\right)+O\left(H^{s}\right) .
$$

To find the formula (24), it is necessary to use $H_{1} D_{\beta}=H_{2} D_{\alpha}$.

From the definition $U=\mathbf{W} \cdot \hat{\mathbf{n}}$, and using basic geometric identities, we can write

$$
\begin{aligned}
\Delta U=\left[\left(\mathbf{W}_{\alpha} \cdot \hat{\mathbf{n}}\right)_{\alpha}+\left(\mathbf{W}_{\beta} \cdot \hat{\mathbf{n}}\right)_{\beta}\right] & +\left(\left(\mathbf{W} \cdot \hat{\mathbf{t}}^{1}\right)\left(-\frac{L}{\sqrt{E}}\right)+\left(\mathbf{W} \cdot \hat{\mathbf{t}}^{2}\right)\left(-\frac{M}{\sqrt{E}}\right)\right)_{\alpha} \\
& +\left(\left(\mathbf{W} \cdot \hat{\mathbf{t}}^{1}\right)\left(-\frac{M}{\sqrt{E}}\right)+\left(\mathbf{W} \cdot \hat{\mathbf{t}}^{2}\right)\left(-\frac{N}{\sqrt{E}}\right)\right)_{\beta} .
\end{aligned}
$$

We use the above formulas to rewrite this to get to the most important parts:

$$
\Delta U=\frac{1}{2} \Lambda\left(\frac{\mu_{\alpha \alpha}+\mu_{\beta \beta}}{\sqrt{E}}\right)-\left(\mathbf{W} \cdot \hat{\mathbf{t}}^{1}\right)\left(\frac{L_{\alpha}+M_{\beta}}{\sqrt{E}}\right)-\left(\mathbf{W} \cdot \hat{\mathbf{t}}^{2}\right)\left(\frac{M_{\alpha}+N_{\beta}}{\sqrt{E}}\right)+O\left(H^{s}\right) .
$$

We now get from (15)

$$
\begin{aligned}
\kappa_{t}=\frac{1}{4 E} \Lambda\left(\frac{\mu_{\alpha \alpha}+\mu_{\beta \beta}}{\sqrt{E}}\right)-\frac{\left(\mathbf{W} \cdot \hat{\mathbf{t}}^{1}\right)}{\sqrt{E}}\left(\frac{L_{\alpha}+M_{\beta}}{2 E}\right) & -\frac{\left(\mathbf{W} \cdot \hat{\mathbf{t}}^{2}\right)}{\sqrt{E}}\left(\frac{M_{\alpha}+N_{\beta}}{2 E}\right) \\
& +\frac{V_{1}}{\sqrt{E}} \kappa_{\alpha}+\frac{V_{2}}{\sqrt{E}} \kappa_{\beta}+O\left(H^{s}\right) .
\end{aligned}
$$

This can be understood better using the following:

$$
\begin{aligned}
& M_{\beta}=-\left(\mathbf{X}_{\beta} \cdot \hat{\mathbf{n}}_{\alpha}\right)_{\beta}=-\mathbf{X}_{\beta} \cdot \hat{\mathbf{n}}_{\alpha \beta}+O\left(H^{s}\right)=-\left(\mathbf{X}_{\beta} \cdot \hat{\mathbf{n}}_{\beta}\right)_{\alpha}+O\left(H^{s}\right)=N_{\alpha}+O\left(H^{s}\right), \\
& M_{\alpha}=-\left(\mathbf{X}_{\alpha} \cdot \hat{\mathbf{n}}_{\beta}\right)_{\alpha}=-\mathbf{X}_{\alpha} \cdot \hat{\mathbf{n}}_{\alpha \beta}+O\left(H^{s}\right)=-\left(\mathbf{X}_{\alpha} \cdot \hat{\mathbf{n}}_{\alpha}\right)_{\beta}+O\left(H^{s}\right)=L_{\beta}+O\left(H^{s}\right) .
\end{aligned}
$$

The previous $\kappa_{t}$ equation can then be written

$$
\kappa_{t}=\frac{1}{4 E} \Lambda\left(\frac{\mu_{\alpha \alpha}+\mu_{\beta \beta}}{\sqrt{E}}\right)+\frac{V_{1}-\mathbf{W} \cdot \hat{\mathbf{t}}^{1}}{\sqrt{E}} \kappa_{\alpha}+\frac{V_{2}-\mathbf{W} \cdot \hat{\mathbf{t}}^{2}}{\sqrt{E}} \kappa_{\beta}+O\left(H^{s}\right) .
$$


Using (12), we see that an exact calculation of $\mu_{\alpha \alpha}$ is

$$
\begin{aligned}
\mu_{\alpha \alpha}=-2 A_{\nu} \sqrt{E} \mathbf{W} & \cdot \hat{\mathbf{t}}_{\alpha}^{1}-R z_{\alpha \alpha}-\frac{A_{\nu}}{\sqrt{E}} E_{\alpha} \mathbf{W} \cdot \hat{\mathbf{t}}^{1}-2 A_{\nu} \sqrt{E} \mathbf{W}_{\alpha} \cdot \hat{\mathbf{t}}^{1} \\
= & -2 A_{\nu}(\mathbf{W} \cdot \hat{\mathbf{n}}) L-R L h-2 A_{\nu} \sqrt{E} \mathbf{W}_{\alpha} \cdot \hat{\mathbf{t}}^{1} \\
& +2 A_{\nu}\left(\mathbf{W} \cdot \hat{\mathbf{t}}^{2}\right) \frac{E_{\beta}}{2 \sqrt{E}}-\frac{A_{\nu}}{\sqrt{E}} E_{\alpha} \mathbf{W} \cdot \hat{\mathbf{t}}^{1}-\frac{R E_{\alpha} z_{\alpha}}{2 E}+\frac{R E_{\beta} z_{\beta}}{2 E} .
\end{aligned}
$$

We can use the formulas for $\nabla \mathbf{W}$ to rewrite this as

$$
\mu_{\alpha \alpha}=-2 A_{\nu} L U-R L h-A_{\nu} \sqrt{E}\left(-H_{1}\left(\frac{\mu_{\alpha} L}{E}\right)-H_{2}\left(\frac{\mu_{\beta} L}{E}\right)\right)+O\left(H^{s+1}\right) .
$$

Similarly, we have for $\mu_{\beta \beta}$ the equation

$$
\mu_{\beta \beta}=-2 A_{\nu} N U-R N h-A_{\nu} \sqrt{E}\left(-H_{1}\left(\frac{\mu_{\alpha} N}{E}\right)-H_{2}\left(\frac{\mu_{\beta} N}{E}\right)\right)+O\left(H^{s+1}\right) .
$$

Using this with the above, we get

$$
\begin{aligned}
\kappa_{t}=-\Lambda\left(\frac{1}{\sqrt{E}}\left[A_{\nu} U+\frac{R h}{2}\right] \kappa\right)+\left(\frac{V_{1}-\mathbf{W} \cdot \hat{\mathbf{t}}^{1}}{\sqrt{E}}-\frac{A_{\nu} \mu_{\alpha}}{2 E}\right) \kappa_{\alpha} \\
+\left(\frac{V_{2}-\mathbf{W} \cdot \hat{\mathbf{t}}^{2}}{\sqrt{E}}-\frac{A_{\nu} \mu_{\beta}}{2 E}\right) \kappa_{\beta}+O\left(H^{s}\right) .
\end{aligned}
$$

Here, we have made use of the formulas

$$
\Lambda H_{1}=-D_{\alpha}, \quad \Lambda H_{2}=-D_{\beta},
$$

which can be seen simply from the symbols.

2.3. The initial value problem. We have only one step left in rewriting the equations. In the first term on the right-hand side of (31), we pull all but $\kappa$ in front of the operator $\Lambda$. As $\Lambda$ is a first-order derivative operator, this will incur a remainder of the form $O\left(H^{s}\right)$ since $U$ and $h$ are of the form $O\left(H^{s+1}\right)$. That $U=O\left(H^{s+1}\right)$ is proved in Lemma 8 below. So, we make the definitions

$$
\begin{gathered}
k(\vec{\alpha}, t)=\frac{A_{\nu} U+\frac{R h}{2}}{\sqrt{E}} \\
\left(T_{1}, T_{2}\right)=\left(\frac{V_{1}-\mathbf{W} \cdot \hat{\mathbf{t}}^{1}}{\sqrt{E}}-\frac{A_{\nu} \mu_{\alpha}}{2 E}, \frac{V_{2}-\mathbf{W} \cdot \hat{\mathbf{t}}^{2}}{\sqrt{E}}-\frac{A_{\nu} \mu_{\beta}}{2 E}\right) .
\end{gathered}
$$

We finally have our preferred form of the evolution equation for $\kappa$,

$$
\kappa_{t}=-k \Lambda(\kappa)+T_{1} \kappa_{\alpha}+T_{2} \kappa_{\beta}+O\left(H^{s}\right) .
$$

We insist upon a condition on the initial data. The above equation makes clear that the evolution equation for $\kappa$ is parabolic. Thus, if $k<0$, it is an ill-posed parabolic equation, and if $k>0$, it is a well-posed parabolic equation. (The case $k>0$ is sometimes called the "stable case".) We make the following assumption on the initial data, so that we are considering the stable case: we assume there exists a positive constant $\bar{k}$ such that for all $\vec{\alpha}$,

$$
k(\vec{\alpha}, 0)>\bar{k}>0 .
$$


Furthermore, we also need to assume that the initial surface is non-self-intersecting. To that end, we assume there exists a positive constant $\bar{c}$ such that for all unequal $\vec{\alpha}$ and $\vec{\alpha}^{\prime}$,

$$
\frac{\left|\mathbf{X}(\vec{\alpha}, 0)-\mathbf{X}\left(\vec{\alpha}^{\prime}, 0\right)\right|}{\left|\vec{\alpha}-\vec{\alpha}^{\prime}\right|}>\bar{c}>0 .
$$

Since we are looking for smooth solutions, the fact that (35) and (36) are satisfied will ensure that at later times, at least for a positive amount of time, we will have

$$
k(\vec{\alpha}, t)>\bar{k}>0, \quad \frac{\left|\mathbf{X}(\vec{\alpha}, t)-\mathbf{X}\left(\vec{\alpha}^{\prime}, t\right)\right|}{\left|\vec{\alpha}-\vec{\alpha}^{\prime}\right|}>\bar{c}>0 .
$$

3. Estimates. We will perform estimates for $\kappa \in H^{s}$; thus, we will have $\mathbf{X}$ and $E$ in $H^{s+2}$, and $\Lambda(\mu) \in H^{s+1}$. We begin with estimates of the quantities related to $\kappa$, such as $\mu$ and the velocities, before proceeding to an energy estimate. We remind the reader of our remark in the introduction: we do not count the minimal $s$. In all of the following estimates (and in any estimates occurring earlier in this paper), it is assumed that $s$ is "large enough".

3.1. Auxiliary estimates. We begin with a lemma about commutators, and then we give lemmas about the regularity of $E$ and $X$.

Lemma 3. If $f \in H^{s+1}$ and $g \in H^{k}$, then $\left[H_{i}, f\right] g \in H^{\min \{k, s\}+1}$.

Proof. If $k \geq s$, then this is proved in 3 . In the other case, $k<s$, the argument of the same theorem in [3] applies. This proves the lemma.

Lemma 4. If $\mathbf{X} \in H^{\ell}$, then $E \in H^{\ell}$.

Proof. We calculate $\Delta E$ :

$$
\Delta E=\left(\mathbf{X}_{\beta} \cdot \mathbf{X}_{\beta}\right)_{\alpha \alpha}+\left(\mathbf{X}_{\alpha} \cdot \mathbf{X}_{\alpha}\right)_{\beta \beta} .
$$

A simple calculation shows that this is the same as

$$
\Delta E=\left(\mathbf{X}_{\alpha} \cdot \mathbf{X}_{\beta}\right)_{\alpha \beta}+O\left(H^{\ell-2}\right) .
$$

Since the isothermal parameterization requires $\mathbf{X}_{\alpha} \cdot \mathbf{X}_{\beta}=0$, the result follows.

Lemma 5. If $\kappa \in H^{s}$ and $\mathbf{X} \in H^{s+1}$, and $\mathbf{X}$ is parameterized according to (2), then $\mathbf{X} \in H^{s+2}$.

Proof. To start, since we have $\mathbf{X} \in H^{s+1}$, we have $E \in H^{s+1}$. Next, we have the following calculation of derivatives of the normal vector:

$$
\begin{aligned}
\Delta \hat{\mathbf{n}} \cdot \hat{\mathbf{t}}^{1} & =\hat{\mathbf{n}}_{\alpha \alpha} \cdot \hat{\mathbf{t}}^{1}+\hat{\mathbf{n}}_{\beta \beta} \cdot \hat{\mathbf{t}}^{1} \\
& =\left(\hat{\mathbf{n}}_{\alpha} \cdot \hat{\mathbf{t}}^{1}\right)_{\alpha}+\left(\hat{\mathbf{n}}_{\beta} \cdot \hat{\mathbf{t}}^{2}\right)_{\alpha}-\left(\left(\hat{\mathbf{n}}_{\alpha} \cdot \hat{\mathbf{t}}^{2}\right)_{\beta}-\left(\hat{\mathbf{n}}_{\beta} \cdot \hat{\mathbf{t}}^{1}\right)_{\beta}\right)+O\left(H^{s-1}\right) \\
& =-\left(\frac{L}{\sqrt{E}}\right)_{\alpha}-\left(\frac{N}{\sqrt{E}}\right)_{\alpha}+\left(\left(\frac{M}{\sqrt{E}}\right)_{\beta}-\left(\frac{M}{\sqrt{E}}\right)_{\beta}\right)+O\left(H^{s-1}\right) \\
& =-(2 \sqrt{E} \kappa)_{\alpha}+O\left(H^{s-1}\right) .
\end{aligned}
$$

Similar calculations for the other components of $\Delta \hat{\mathbf{n}}$ indicate that $\Delta \hat{\mathbf{n}}$ is in $H^{s-1}$. Thus, $\hat{\mathbf{n}} \in H^{s+1}$. This is a gain of one derivative. This in turn implies a gain of one derivative 
in each of $L, M$, and $N$ (so that they are in $H^{s}$ ). Taking derivatives of $\hat{\mathbf{t}}^{1}$ and $\hat{\mathbf{t}}^{2}$, in view of equations such as

$$
\hat{\mathbf{t}}_{\alpha}^{1}=\frac{L}{\sqrt{E}} \hat{\mathbf{n}}-\frac{E_{\beta}}{2 E} \hat{\mathbf{t}}^{2},
$$

and using $\mathbf{X}_{\alpha}=\sqrt{E} \hat{\mathbf{t}}^{1}$ and $\mathbf{X}_{\beta}=\sqrt{E} \hat{\mathbf{t}}^{2}$, we also see a gain of one derivative for $\mathbf{X}_{\alpha}$ and $\mathbf{X}_{\beta}$. Integrating, we find that $\mathbf{X}$ is in $H^{s+2}$.

Lemma 6. If $\mathbf{X} \in H^{s+2}$, then $\mu$ is well-defined and $\mu \in H^{0}$.

REMark. We do not prove this lemma here. This lemma says that (11), which can be viewed as an integral equation for $\mu$, is solvable, with the solution in $L^{2}$. This is discussed in [19] and [4. The next lemma tells us about the higher regularity of $\mu$.

Lemma 7. If $\mathbf{X} \in H^{s+2}$, then $\mu \in H^{s+2}$.

Proof. Letting $g=\mu_{\alpha} \mathbf{X}_{\beta}-\mu_{\beta} \mathbf{X}_{\alpha}$, and letting a prime after a variable indicate that it is evaluated at $\vec{\alpha}^{\prime}$ (and thus unprimed variables are evaluated at $\vec{\alpha}$ ), we write

$$
\mathbf{W}(\vec{\alpha})=-\frac{1}{4 \pi} \iint g^{\prime} \times \frac{\mathbf{X}-\mathbf{X}^{\prime}}{\left|\mathbf{X}-\mathbf{X}^{\prime}\right|^{3}} d \vec{\alpha}^{\prime}
$$

Recalling the definition of the kernel $K$, and denoting the integral operator with kernel $K$ as $\mathcal{K}[\mathbf{X}]$, we write

$$
\begin{aligned}
\mathbf{W}(\vec{\alpha})=\mathcal{K}[\mathbf{X}](g)+H_{1}\left(\frac{g \times \mathbf{X}_{\alpha}}{2 E^{3 / 2}}\right)+H_{2}\left(\frac{g \times \mathbf{X}_{\beta}}{2 E^{3 / 2}}\right) & \\
+G_{11}\left(\frac{g \times \mathbf{X}_{\alpha \alpha}}{2 E^{3 / 2}}-\frac{3 g \times E_{\alpha} X_{\alpha}}{4 E^{5 / 2}}\right)+G_{12} & \left.\frac{g \times \mathbf{X}_{\alpha \beta}}{E^{3 / 2}}-\frac{g \times\left(E_{\alpha} \mathbf{X}_{\beta}+E_{\beta} \mathbf{X}_{\alpha}\right)}{E^{5 / 2}}\right) \\
& +G_{22}\left(\frac{g \times \mathbf{X}_{\beta \beta}}{2 E^{3 / 2}}-\frac{3 g \times E_{\beta} X_{\beta}}{4 E^{5 / 2}}\right)
\end{aligned}
$$

Above, we have used the operators $G_{i j}$. For example, $G_{11}$ is defined as the operator with kernel $\frac{\left(\alpha-\alpha^{\prime}\right)^{2}}{\left|\vec{\alpha}-\vec{\alpha}^{\prime}\right|^{3}}$, and the others are similar. These are operators which are smoothing by one derivative, since the kernel can absorb one derivative. These operators are discussed further in [3].

We will let $\mathbf{G}$ denote the sum of the $G_{i j}$ terms, so that

$$
\mathbf{W}=\mathcal{K}[\mathbf{X}](g)+H_{1}\left(\frac{g \times \mathbf{X}_{\alpha}}{2 E^{3 / 2}}\right)+H_{2}\left(\frac{g \times \mathbf{X}_{\beta}}{2 E^{3 / 2}}\right)+\mathbf{G} .
$$

Since $\mathbf{X}_{\alpha} \times \mathbf{X}_{\beta}=E \hat{\mathbf{n}}$, we can rewrite this as

$$
\mathbf{W}=\mathcal{K}[\mathbf{X}](g)+H_{1}\left(\frac{\mu_{\alpha}}{2 E^{1 / 2}} \hat{\mathbf{n}}\right)+H_{2}\left(\frac{\mu_{\beta}}{2 E^{1 / 2}} \hat{\mathbf{n}}\right)+\mathbf{G} .
$$

We pull the normal vector outside of the Riesz transforms, incurring a commutator. Thus, we have

$$
\mathbf{W}=\mathcal{K}[\mathbf{X}](g)+H_{1}\left(\frac{\mu_{\alpha}}{2 E^{1 / 2}}\right) \hat{\mathbf{n}}+H_{2}\left(\frac{\mu_{\beta}}{2 E^{1 / 2}}\right) \hat{\mathbf{n}}+\mathcal{G},
$$


where $\mathcal{G}$ is the sum of $\mathbf{G}$ and the commutators:

$$
\begin{aligned}
\mathcal{G}=\mathbf{G} & +\left[H_{1}, \hat{\mathbf{n}}\right]\left(\frac{\mu_{\alpha}}{2 E^{1 / 2}}\right)+\left[H_{2}, \hat{\mathbf{n}}\right]\left(\frac{\mu_{\beta}}{2 E^{1 / 2}}\right) \\
= & G_{11}\left(\frac{g \times \mathbf{X}_{\alpha \alpha}}{2 E^{3 / 2}}-\frac{3 g \times E_{\alpha} X_{\alpha}}{4 E^{5 / 2}}\right)+G_{12}\left(\frac{g \times \mathbf{X}_{\alpha \beta}}{E^{3 / 2}}-\frac{g \times\left(E_{\alpha} \mathbf{X}_{\beta}+E_{\beta} \mathbf{X}_{\alpha}\right)}{E^{5 / 2}}\right) \\
& +G_{22}\left(\frac{g \times \mathbf{X}_{\beta \beta}}{2 E^{3 / 2}}-\frac{3 g \times E_{\beta} X_{\beta}}{4 E^{5 / 2}}\right)+\left[H_{1}, \hat{\mathbf{n}}\right]\left(\frac{\mu_{\alpha}}{2 E^{1 / 2}}\right)+\left[H_{2}, \hat{\mathbf{n}}\right]\left(\frac{\mu_{\beta}}{2 E^{1 / 2}}\right) .
\end{aligned}
$$

For $\mathbf{X} \in H^{s+2}$ and $\nabla_{\vec{\alpha}} \mu \in H^{k}$, we have that $E \in H^{s+2}$ and $g \in H^{\min \{k, s+1\}}$. Since the operators $G_{i j}$ gain one derivative over the function being acted on, and that function is in $H^{\min \{k, s\}}$, we see that $\mathbf{G} \in H^{\min \{k, s\}+1}$. For the commutators, we use Lemma 3 with $f=\hat{\mathbf{n}} \in H^{s+1}$. We conclude that the commutators are in $H^{\sigma}$ with $\sigma=\min \{\min \{k, s+2\}, s\}+1$. This clearly is the same as saying the commutators are in $H^{\min \{k, s\}+1}$. Finally, this implies that $\mathcal{G} \in H^{\min \{k, s\}+1}$.

We now turn to the question of which space $\mathcal{K}[\mathbf{X}](g)$ is in when $\mathbf{X} \in H^{s+2}$ and $\nabla_{\vec{\alpha}} \mu \in H^{k}$. As in the proof of Lemma 3, we rely on theorems proved in [3. In particular, for $k \geq s-1 / 2$, a result of [3] implies that $\mathcal{K}[\mathbf{X}](g) \in H^{s+1}$. For $k<s-1 / 2$, the argument in the proof of the same theorem implies that $\mathcal{K}[\mathbf{X}](g) \in H^{k+1}$. (The argument would actually provide higher regularity than this, but this is sufficient for the present purposes.) Thus, no matter which case we are in, we have $\mathcal{K}[\mathbf{X}](g) \in H^{\min \{k, s\}+1}$.

Now, for the regularity of $\mu$, we use (40) together with Lemma 6 and (12), (13). Lemma 6 tells us that $\mu \in H^{0}$, and thus $\nabla_{\vec{\alpha}} \mu \in H^{-1}$. The above calculations imply that $\mathbf{W} \cdot \hat{\mathbf{t}}^{i}$ is then in $H^{0}$. The formulas (12) and (13) in turn tell us that $\mu_{\alpha}$ and $\mu_{\beta}$ are in $H^{0}$. This argument can be repeated; the highest regularity is determined by the regularity of $\mathbf{X}$. In particular, the above calculations will apply until $k=s$. We are able to perform this argument for the final time when $\nabla_{\vec{\alpha}} \mu \in H^{s}$. We find that $\mathbf{W} \cdot \hat{\mathbf{t}}^{i}$ is in $H^{s+1}$. Finally, we conclude that $\nabla_{\vec{\alpha}} \mu \in H^{s+1}$.

Lemma 8. We have the following estimates for the velocities:

$$
\begin{aligned}
\|\mathbf{W}\|_{s+1} & \leq C\left(1+\|\kappa\|_{s}\right)^{p}, \\
\left\|V_{i}\right\|_{s+1} & \leq C\left(1+\|\kappa\|_{s}\right)^{p} .
\end{aligned}
$$

The estimate on $\mathbf{W}$ follows immediately from formulas (20)-(24) and from Lemma 6 . For the $V_{i}$, we rewrite the equations (3), (4) as

$$
\begin{gathered}
\left(\frac{V_{1}}{\sqrt{E}}\right)_{\alpha}-\left(\frac{V_{2}}{\sqrt{E}}\right)_{\beta}=\frac{U(L-N)}{E}, \\
\left(\frac{V_{1}}{\sqrt{E}}\right)_{\beta}+\left(\frac{V_{2}}{\sqrt{E}}\right)_{\alpha}=\frac{2 U M}{E} .
\end{gathered}
$$

Taking an $\alpha$-derivative of (42) and adding a $\beta$-derivative of (43), we get

$$
V_{1}=\sqrt{E} \Delta^{-1}\left(\left(\frac{U(L-N)}{E}\right)_{\alpha}+\left(\frac{2 U M}{E}\right)_{\beta}\right) .
$$


We have a similar expression for $V_{2}$ :

$$
V_{2}=\sqrt{E} \Delta^{-1}\left(\left(\frac{2 U M}{E}\right)_{\alpha}-\left(\frac{U(L-N)}{E}\right)_{\beta}\right) .
$$

For the quantities on the right-hand side, $U=\mathbf{W} \cdot \hat{\mathbf{n}}$ is in $H^{s+1}$, and $L, M$, and $N$ are in $H^{s}$. Furthermore, $E$ is in $H^{s+2}$. Therefore, we see that each of the $V_{i}$ can be bounded in $H^{s+1}$.

3.2. The energy estimate. We now define an energy functional; it is in fact just the square of the $H^{s}$ norm of $\kappa$, plus the square of a norm of $\mathbf{X}$ (below the highest regularity) included for technical reasons. We have

$$
E^{s}(t)=\frac{1}{2}\|\kappa\|_{s}^{2}+\frac{1}{2}\|\mathbf{X}\|_{s+1}^{2}
$$

Lemma 9. The energy $E^{s}$ satisfies the following differential inequality:

$$
\frac{d E^{s}}{d t} \leq O\left(H^{0}\right) .
$$

We take the time derivative of the energy. The time derivative of $\mathbf{X}$ is $O\left(H^{s+1}\right)$; this is proved in Lemma 8 above. Furthermore, letting $\sigma$ be a multi-index of order $s-1$ or less, we have that $D^{\sigma} \kappa_{t}$ is at least $O\left(H^{0}\right)$. This implies that

$$
\frac{d E^{s}}{d t}=\sum_{|\sigma|=s} \int\left(D^{\sigma} \kappa\right)\left(D^{\sigma} \kappa_{t}\right) d \vec{\alpha}+O\left(H^{0}\right)
$$

Using (34), and keeping in mind Lemma 8, we see that we can write $D^{\sigma} \kappa_{t}$ as

$$
D^{\sigma} \kappa_{t}=-k \Lambda\left(D^{\sigma} \kappa\right)+T_{1} D^{\sigma} \kappa_{\alpha}+T_{2} D^{\sigma} \kappa_{\beta}+O\left(H^{0}\right) .
$$

Plugging this in, we have

$$
\frac{d E^{s}}{d t}=-\sum_{|\sigma|=s} \int\left(D^{\sigma} \kappa\right)\left(k \Lambda\left(D^{\sigma} \kappa\right)\right)+T_{1}\left(D^{\sigma} \kappa\right)\left(D^{\sigma} \kappa\right)_{\alpha}+T_{2}\left(D^{\sigma} \kappa\right)\left(D^{\sigma} \kappa\right)_{\beta} d \vec{\alpha}+O\left(H^{0}\right) .
$$

The transport terms are handled by the usual integration by parts. We can then rewrite this as

$$
\begin{gathered}
\frac{d E^{s}}{d t}=-\sum_{|\sigma|=s} \int\left(\sqrt{k} D^{\sigma} \kappa\right)\left(\sqrt{k} \Lambda\left(D^{\sigma} \kappa\right)\right) d \vec{\alpha}+O\left(H^{0}\right) \\
=-\sum_{|\sigma|=s} \int\left(\sqrt{k} D^{\sigma} \kappa\right)\left(\Lambda\left(\sqrt{k} D^{\sigma} \kappa\right)\right) d \vec{\alpha}+O\left(H^{0}\right) \\
=-\sum_{|\sigma|=s} \int\left(\Lambda^{1 / 2}\left(\sqrt{k} D^{\sigma} \kappa\right)\right)^{2} d \vec{\alpha}+O\left(H^{0}\right) .
\end{gathered}
$$

We have used (35) in order to take the square root of $k$. In moving $\sqrt{k}$ inside $\Lambda$ (and thus inside the Riesz transforms), we have used Lemma 3 . Since the terms being summed over on the right-hand side of the last equation of (46) are nonpositive, this proves the theorem. 
4. Conclusion. The existence of solutions (in the stable case) now follows from standard arguments. In particular, the method used in [1] to prove well-posedness of the vortex sheet with surface tension or in [16] to prove well-posedness of the Navier-Stokes equations would now apply.

The method is to first introduce mollifiers to the right-hand side of the evolution equation. Then, Picard's theorem for ODEs on a Banach space implies the existence of solutions to the mollified equation for a (very) short interval of time. The mollifiers can be introduced in such a way that the energy estimate holds for the mollified system, uniformly in the mollification parameter, so that the mollified solutions are uniformly bounded on a common time interval (this makes use of a continuation theorem for autonomous ODEs on a Banach space). We are also able to show, by an estimate similar to the energy estimate, that the mollified solutions form a Cauchy sequence in a low norm. Together with the uniform bound in the high norm, this allows us to pass to the limit as the mollification parameter tends to zero, proving the existence of solutions to the nonmollified problem. Similar estimates then allow us to prove uniqueness, continuous dependence, and regularity.

We expect, since it has been proved for a number of other Darcy flows, including the two-phase problem in the absence of surface tension [18, that it should be possible to prove the existence for all time of small solutions. This question will be taken up in a future work, rather than addressing it here.

Since the solution is smooth, and (35) holds initially, it will continue to hold for at least a positive amount of time. This amount of time would depend on the initial conditions.

The energy estimates of the previous section could also be carried out in the case in which surface tension is present. Then, going from the energy estimates to the existence proof is a bit more delicate, but is still possible. For details of such an existence argument, the reader should consult $[3$.

We conclude the paper by stating the theorem we have proved:

Theorem 10. Let $s$ be a positive integer, large enough. Let initial data $\kappa_{0} \in H^{s}$ be given such that the associated surface $\mathbf{X}$ is parameterized by (2) and satisfies (36) and the associated quantity $k$ satisfies (35). There exists a unique solution of the initial value problem given by (34) such that $\kappa(\vec{\alpha}, 0)=\kappa_{0}(\vec{\alpha})$. There is a time $T>0$ such that this solution is in $C\left([0, T] ; H^{s}\right)$, and until time $T$, the conditions (37) are satisfied. $T$ depends on $\bar{k}, \bar{c}, s$, and $\kappa_{0}$.

We remark that we can also prove a continous dependence theorem. Furthermore, we remark that if the initial surface does not admit a global isothermal parameterization, a modification of the argument above in which we use a finite number of overlapping coordinate patches could be used.

\section{REFERENCES}

[1] Ambrose, D.M. Well-posedness of vortex sheets with surface tension. SIAM J. Math. Anal. 35 (2003), 211-244. MR2001473 (2005g:76006)

[2] Ambrose, D.M. Well-posedness of two-phase Hele-Shaw flow without surface tension. European J. Appl. Math. 15 (2004) 597-607. MR2128613(2005m:76065) 
[3] Ambrose, D.M.; Masmoudi, N. Well-posedness of 3D vortex sheets with surface tension. (2006) Submitted.

[4] Ambrose, D.M.; Masmoudi, N. The zero surface tension limit of three-dimensional water waves. (2006) In preparation.

[5] Baker, G.; Meiron, D.; Orszag, S. Generalized vortex methods for free-surface flow problems. J. Fluid Mech. 123 (1982), 477-501. MR0687014 (84a:76002)

[6] Caflisch, R.E.; Li, X.-F. Lagrangian theory for 3D vortex sheets with axial or helical symmetry. Transport Theory Statist. Phys. 21 (1992), 559-578. MR1194461 (93h:76014)

[7] Chen, X.; Friedman, A. A free boundary problem for an elliptic-hyperbolic system: an application to tumor growth. SIAM J. Math. Anal. 35 (2003) 974-986. MR2049029 (2005f:35333)

[8] Cordoba, D.; Gancedo, F. Contour dynamics of incompressible 3-D fluids in a porous medium with different densities. (2006) Preprint.

[9] Escher, J.; Simonett, G. Classical solutions of multidimensional Hele-Shaw models. SIAM J. Math. Anal. 28 (1997) 1028-1047. MR1466667(98i:35213)

[10] Escher, J.; Simonett, G. A center manifold analysis for the Mullins-Sekerka model. J. Differential Equations 143 (1998), 267-292. MR1607952 (98m:35228)

[11] Friedman, A. Time dependent free boundary problems. SIAM Rev. 21 (1979) 213-221. MR0524512 (81g:76097)

[12] Friedman, A. Free boundary problems with surface tension conditions. Nonlinear Analysis 63 (2005) 666-671. MR2188139 (2006f:35301)

[13] Friedman, A.; Reitich, F. Nonlinear stability of a quasi-static Stefan problem with surface tension: A continuation approach. Ann. Scuola Norm. Sup. Pisa Cl. Sci. (4) 30 (2001), 341-403. MR1895715 (2003e:35326)

[14] Hou, T.; Lowengrub, J.; Shelley, M. Removing the stiffness from interfacial flows with surface tension. J. Comput. Phys. 114 (1994), 312-338. MR1294935 (95e:76069)

[15] Hou, T.; Lowengrub, J.; Shelley, M. The long-time motion of vortex sheets with surface tension. Phys. Fluids 9 (1997), 1933-1954. MR1455083(98d:76033)

[16] Majda, A.; Bertozzi, A. Vorticity and Incompressible Flow. Cambridge University Press, Cambridge, UK, 2002. MR1867882(2003a:76002)

[17] Saffman, P.G. Vortex dynamics. Cambridge University Press, Cambridge, UK, 1992. MR1217252 (94c:76015)

[18] Siegel, M.; Caflisch, R.; Howison, S. Global existence, singular solutions, and ill-posedness for the Muskat problem. Comm. Pure Appl. Math. 57 (2004), 1374-1411. MR2070208

[19] Wu, S. Well-posedness in Sobolev spaces of the full water wave problem in 3-D. J. Amer. Math. Soc. 12 (1999), 445-495. MR1641609 (2001m:76019) 